\title{
Factors Affecting the Catalytic Hydrogenation of $p$-Nitrophenol by Nano Nickel Supported on Egyptian Kaolin
}

\author{
M.M. Selim* and N.A. Fathy
}

Physical Chemistry Department, National Research Center, Cairo, Egypt.

\begin{abstract}
7 HE LIQUID-PHASE catalytic hydrogenation of $p$-nitrophenol to $p$-aminophenol was studied over kaolin supported nano-nickel catalysts prepared by chemical reduction by hydrazine hydrate. It was found that the $5 \% \mathrm{Ni}-\mathrm{K}$ catalyst has a superior catalytic activity corresponding to the other nickel contents, and hence it was chosen for determining the optimum conditions of such reaction. The effect of catalyst loading, $p$-nitrophenol concentration, $\mathrm{NaOH}$ and $\mathrm{NH}_{4} \mathrm{OH}$ concentrations on the catalytic activity of nano-nickel catalyst was investigated at $80^{\circ} \mathrm{C}$. It was assessed that the catalytic efficiency of the catalyst was enhanced considerably by changing in $\mathrm{NaOH}$ and $\mathrm{NH}_{4} \mathrm{OH}$ concentrations during hydrogenation reaction.
\end{abstract}

Keywords: Nano-nickel catalyst, Egyptian kaolin support and Hydrogenation.

In the recent years, nanoparticle synthesis has been widely investigated because of its many unique characteristics in physical and chemical properties. Nanosized nickel catalysts have received increasing attention since such materials exhibit many fascinating chemical and physical characteristics and potential technological applications such as catalysts, batteries and in super alloys ${ }^{(1,2)}$. Several papers have been published on the preparation ${ }^{(1-5)}$ and applications ${ }^{(6-9)}$ of nano-sized nickel catalysts. It is reported that nano-sized nickel shows better catalytic properties in the catalytic hydrogenation or dehydrogenation reaction, and thermal stability were better than those of a commercial Raney nickel ${ }^{(1-9)}$.

Since p-aminophenol (PAP) compounds are of great commercial importance as an intermediate for the preparation of analgesic and antipyretic drugs ${ }^{(10-13)}$. So, there are many methods used in the preparation of PAP from p-nitrophenol (PNP) including metal-acid reduction, catalytic reduction, electrolytic reduction, homogeneous and heterogeneous catalytic transfer reduction and direct catalytic reduction reactions ${ }^{(4)}$. Among these methods, direct catalytic reduction is widely used for the production of PAP because it represents the greener and effective technology ${ }^{(13)}$. Earlier many studies have reported that the liquid phase hydrogenation in presence of a solid catalyst is a simple way to improve the conversion and selectivity ${ }^{(4,5,14,15)}$. Thus various metal catalysts, for example, Raney nickel, nano-sized nickel, and noble metals ( $\mathrm{Pt}, \mathrm{Pd}, \mathrm{Ru}, \mathrm{Ag} . . .$, etc.), have

\footnotetext{
*Corresponding author E-mail:mmaselim@yahoo.com
} 
been investigated extensively ${ }^{(14,15)}$. Several studies have been carried out on preparation of nano-nickel catalysts supported on different low-cost and available supports using direct chemical reduction method ${ }^{(4,5,8,10)}$.

According to the abovementioned literature, the direct catalytic hydrogenation of p-nitrophenol to p-aminophenol compounds over nano-sized nickel supported on kaolin has not been reported yet. Therefore, in this research, we have tried to synthesize PAP from p-nitrophenol over nano-sized nickel catalysts supported on kaolin. The nano-sized nickel catalysts are prepared by a chemical reduction method. Also, the catalytic performance of nano-sized nickel supported on Egyptian's kaolin for hydrogenation of p-nitrophenol in liquid phase under different conditions such as: weight percent of nickel, catalyst dose, initial concentration of PNP; $\mathrm{NaOH}$ and $\mathrm{NH}_{4} \mathrm{OH}$ concentrations were studied.

\section{Materials}

\section{Experimental}

All chemicals used were analytical grade (AR); $\mathrm{Ni}\left(\mathrm{NO}_{3}\right)_{2} \cdot 6 \mathrm{H}_{2} \mathrm{O}, \mathrm{NaCl}$, $\mathrm{NaOH}$, hydrazine hydrated, p-nitrophenol, methanol, and distilled water, and natural Egyptian kaolin.

\section{Preparation of nano-sized nickel over kaolin}

Kaolin supported nano-nickel catalysts were prepared by the direct reduction as follows. Firstly, $100 \mathrm{~g}$ of kaolin was washed gently with hot distilled water followed by soaking in $1 \mathrm{~L}$ of $1 \mathrm{M} \mathrm{NaCl}$ solution for $24 \mathrm{hr}$ at $30^{\circ} \mathrm{C}$, to remove any undesirable components, decanted, washed and then dried in an air oven overnight at $100^{\circ} \mathrm{C}$. Secondly, nickel was loaded on kaolin by the means wetness impregnation method at different contents; 2.5, 5 and $10 \mathrm{wt} \%$, and denoted as $2.5 \mathrm{Ni}-\mathrm{K}, 5 \mathrm{Ni}-\mathrm{K}$ and $10 \mathrm{Ni}-\mathrm{K}$, respectively. Direct reduction was carried out by hydrazine hydrate in order to obtain the metallic form of nickel. The reaction was allowed to continue until no bubbles were observed. The product (grey-black) was then washed with anhydrous ethanol and then isolated by decantation.

\section{Liquid-phase reduction reaction}

This reaction was preceded by dissolving PNP in an appropriate amount of methanol, then hydrazine hydrate was added and afterwards the mixture was heated at $80^{\circ} \mathrm{C}$. The catalyst was added to the heated solution and then recorded the time required to attain $100 \%$ conversion of PNP into PAP which measures the catalytic activity. In this study, hydrogenation of p-nitrophenol into paminophenol was performed on $5 \mathrm{wt} \%$ of Ni catalyst supported onto kaolin as shown in Fig. $1^{(4)}$. Hydrazine is a normal reducer; its reductive ability varies according to the $\mathrm{pH}$ value of the solution. In acid medium, $\mathrm{N}_{2} \mathrm{H}_{4}$ is easily oxidized to $\mathrm{NH}_{3}$, whereas in a basic medium, it can be easily oxidized to $\mathrm{N}_{2}$, so it is possible to reduce nickel ions in basic medium. hydrazine is added to the solution containing nickel nitrate at alkaline medium and $80^{\circ} \mathrm{C}$ to enhance the reaction rate. The reaction equation between nickel and hydrazine is given:

$$
2 \mathrm{Ni}_{2}{ }^{+}+\mathrm{N}_{2} \mathrm{H}_{4}+4 \mathrm{OH}^{-}=2 \mathrm{Ni}+\mathrm{N}_{2} \uparrow+4 \mathrm{H}_{2} \mathrm{O}(1)
$$

Egypt. J. Chem. 55, No. 6 (2012) 
<smiles>O=[N+]([O-])c1ccc(O)cc1</smiles>

\section{5wt $\%$ supported $\mathrm{Ni}$ Catalyst}

Hydrazine

p-nitrophenol<smiles>Nc1ccc(O)cc1</smiles>

p-aminophenol

Fig. 1. Schematic diagram for conversion of p-nitrophenol to p-amino phenol.

In addition, Selim et $a l .{ }^{(4)}$ proposed the mechanism that takes place during hydrogenation process using self-change in color. They observed a change from yellow color of PNP into green color (intermediate A) followed by a discharge of all colors (colorless) which accompanied with $100 \%$ conversion of PNP to PAP. The mechanism of this reaction is shown in Fig. 2. Since the supported nickel catalyst acts as a bi-functional catalyst, it will decompose hydrazine into $\mathrm{H}_{2}$ and $\mathrm{N}_{2}$. Furthermore, the nickel acts as a catalyst for the hydrogenation of $\mathrm{p}$ nitrophenol using the nascent hydrogen produced from previous step.

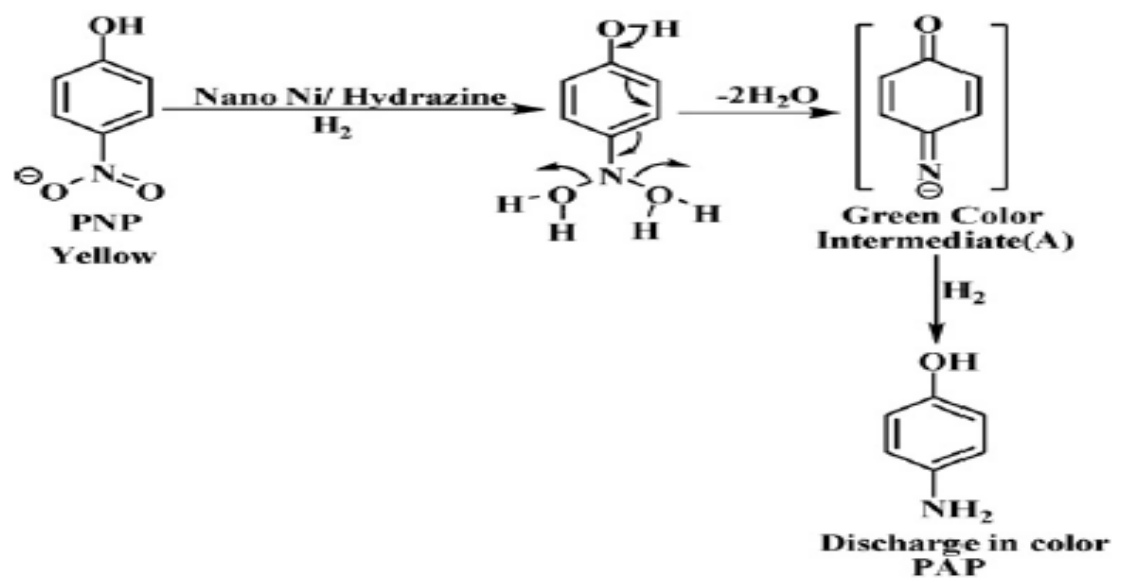

Fig. 2. Schematic mechanism for conversion of p-nitrophenol to p-amino phenol. 
Characterization of the nanocatalyst

$\mathrm{X}$-ray diffraction patterns for the $10 \mathrm{Ni}-\mathrm{K}$ catalyst before and after reduction process were determined using Bruker D8 advance instrument with $\mathrm{CuK} \alpha$ target with secondly monochromate $40 \mathrm{Kv}, 40 \mathrm{~mA}$.

\section{Factors affecting the reduction reaction}

In order to investigate the factors affecting the catalytic performance of $\mathrm{Ni}-\mathrm{K}$ prepared catalyst, the reaction variables such as catalyst dose $(0.2,0.5,1$ and $2 \mathrm{~g})$, initial concentration of PNP $(0.25,0.5,1$ and $5 \mathrm{~g} / \mathrm{L}), \mathrm{NaOH}$ concentration $(0.15$, $0.30,0.70,1.0,1.6$ and $2.0 \mathrm{~g} / 10 \mathrm{ml}$ methanol) and ammonia concentration $(0,3$, 10,15 and $25 \%$ ) were studied.

\section{Results and Discussion}

\section{$X$ - ray diffraction results}

Figure 3 ( $a$ and $b$ ) illustrates the data obtained from XRD-technique. Figure 3a shows the XRD-data of the impregnated kaolin with nickel nitrate. It can be seen that the lines located at $2 \theta=30$ and $47^{\circ}$ are related to crystalline nickel nitrate on kaolin. Figure $3 \mathrm{~b}$ indicates the patterns of crystalline reduced nickel on kaolin with disappearing of the lines regarding to crystalline nickel nitrate, indicating complete reduction of nickel nitrate to metallic nickel with lines at $2 \theta$ $=44.5$ and 51.85 .

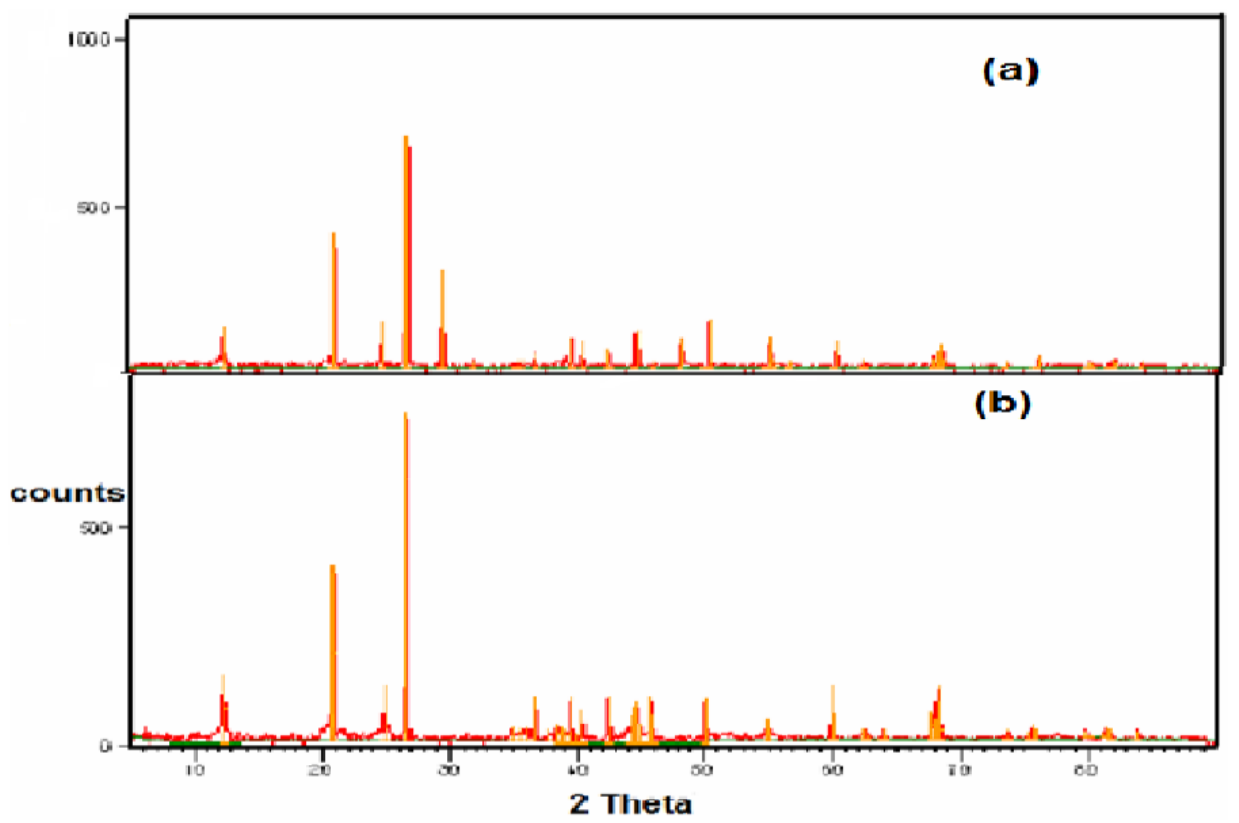

Fig. 3. XRD patterns of (a) $\mathrm{Ni}\left(\mathrm{NO}_{3}\right)_{2}$ and (b) Ni-metal supported on kaolin.

Egypt. J. Chem. 55, No. 6 (2012) 
Effect of nickel percent on the activity of catalyst

It is well-known that the change in nickel percent would affect the catalytic activity of prepared catalyst supported on kaolin. Figure 4 shows the effect of nickel weight percent on the hydrogenation of p-nitrophenol (PNP) into paminophenol (PAP) within ten runs of reduction process. It was observed that the prepared 5\% Ni-nanoparticles exhibited better activity (100\%) for the conversion of PNP to PAP within $440 \mathrm{sec}$. Hence, it can be concluded that the catalytic activity of $5 \% \mathrm{Ni}$ catalyst is more efficient than that of others.

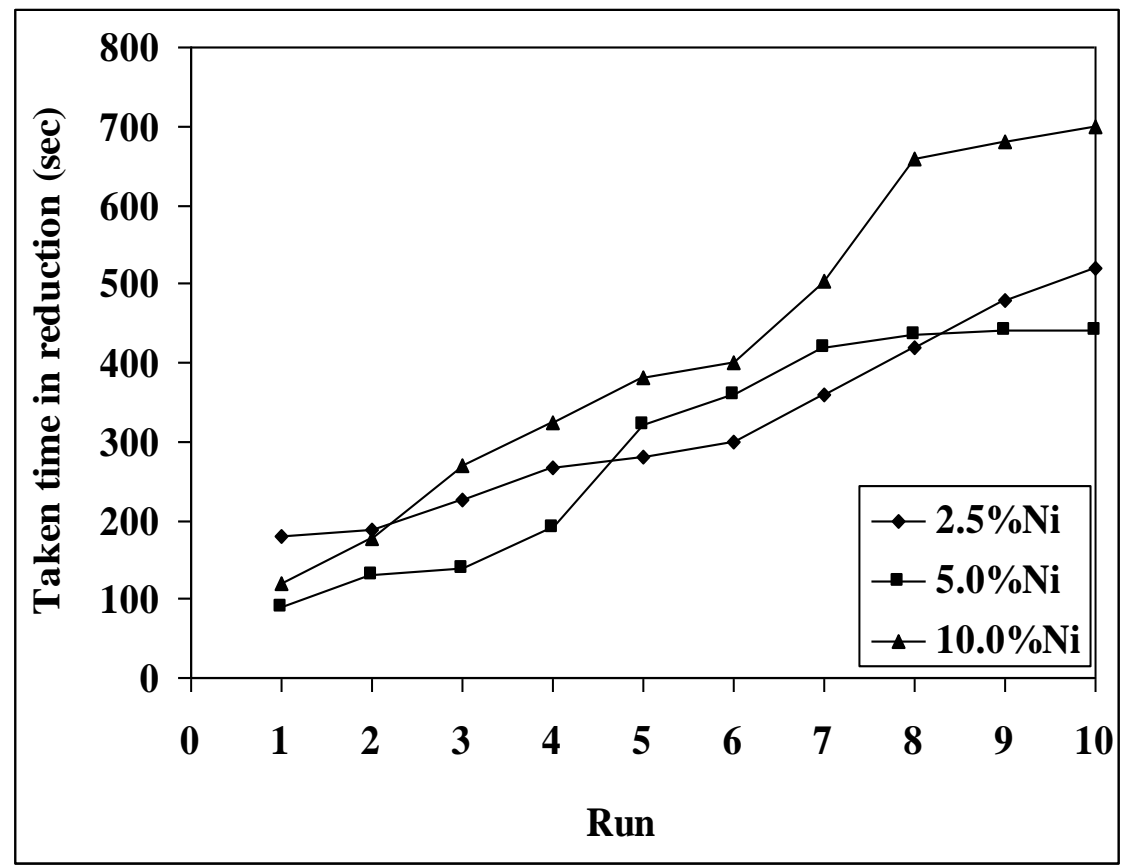

Fig. 4. Effect of Ni content on the reduction of PNP into PAP.

\section{Catalytic performance study}

The catalytic performance of the prepared catalyst was determined in relation to the change in the initial concentration of p-nitrophenol $(0.25-5 \mathrm{~g} / \mathrm{ml})$. Figure 5 shows the catalytic activity of the prepared catalysts as a function of PNP initial concentration; and the complete conversion of PNP into PAP compounds was detected at the time in which the yellow color of the mixture turns into colorless. It can be seen from Fig. 5, clearly that the increase from 2.5 to $5 \%$ of nickel on the support increases the activity, i.e. it decreases the time needed for the complete reduction of PNP. Further increase from 5 to $10 \%$ of Ni on the support produces catalyst with a relatively lower activity. This behavior may be attributed to the accumulation of nickel particles and consequently inhibits the activity of catalyst (10Ni-K). 


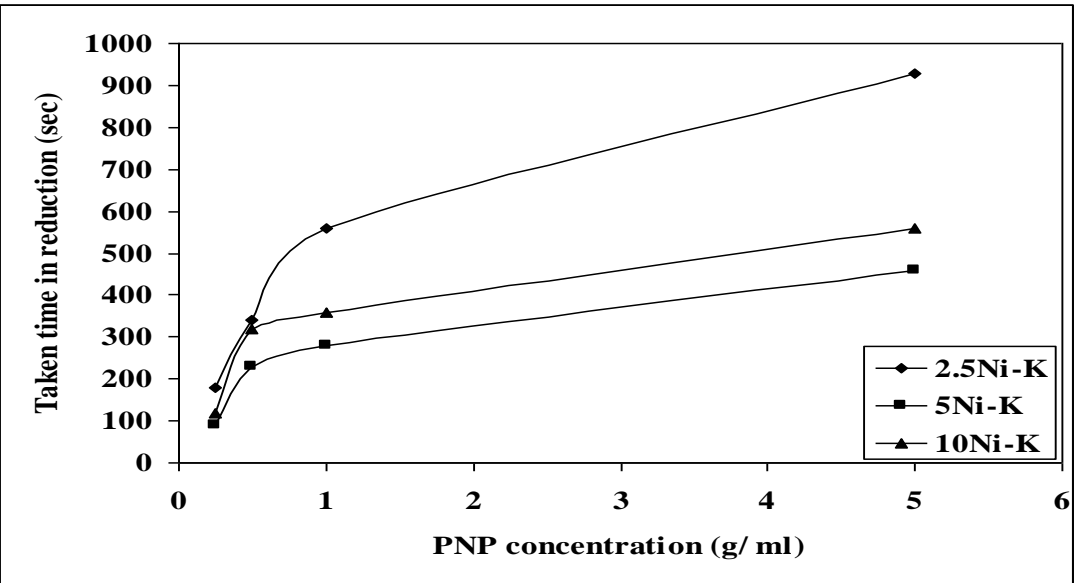

Fig. 5. Catalytic activity of the prepared catalysts in relation to initial concentration of PNP.

Effect of catalyst dose

The effect of catalyst dose, i.e. $5 \mathrm{Ni}-\mathrm{K}$, on the reduction process of PNP is shown in Fig. 6. It is clear from this figure that, the increase in catalyst dose from 0.2 up to $1 \mathrm{~g}$ leads to gradual decrease in the time needed for complete reduction of p-nitrophenol into p-aminophenol. It can be seen that $0.2 \mathrm{~g}$ of $5 \mathrm{Ni}-\mathrm{K}$ catalyst reduces PNP completely into PAP within $1800 \mathrm{sec}(30 \mathrm{~min})$, further increase to $1 \mathrm{~g}$ leads to complete reduction within $220 \mathrm{sec}$ (3.66 min). However, above this amount of catalyst, did not show significant change and the time required to attain complete conversion becomes steady. The optimum catalyst weight was chosen at $1 \mathrm{~g}$ of $5 \mathrm{Ni}-\mathrm{K}$ for reduction of PNP.

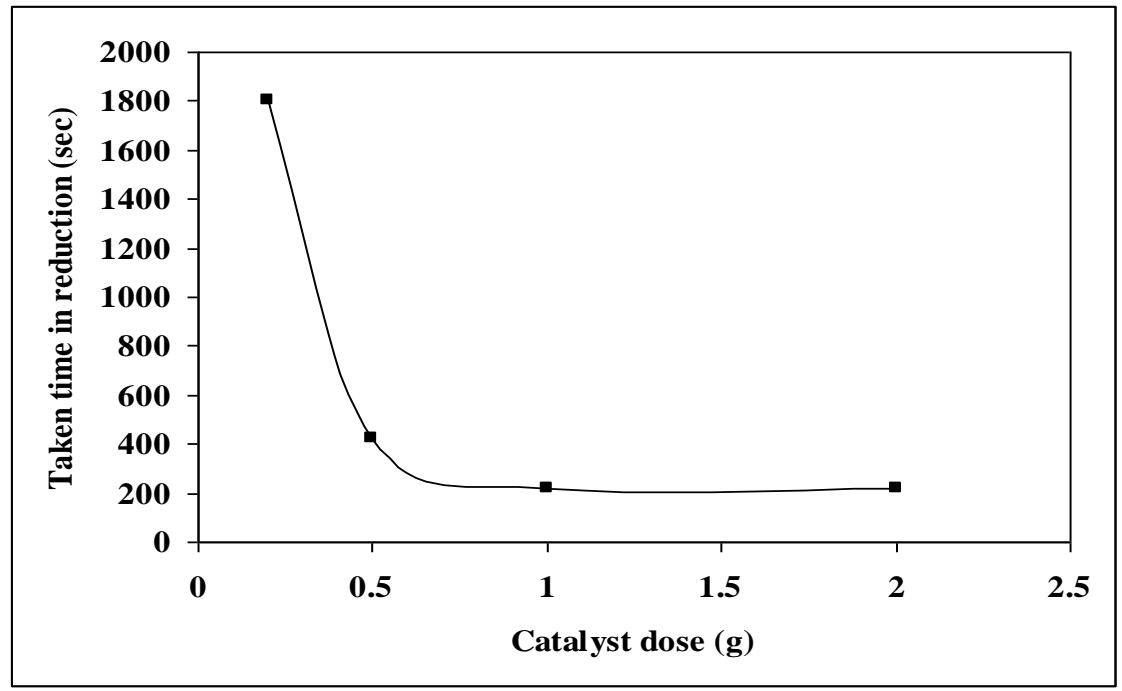

Fig. 6. Effect of catalyst dose on the conversion of PNP into PAP.

Egypt. J. Chem. 55, No. 6 (2012) 


\section{Effect of $\mathrm{NaOH}$ concentration}

It is important to explore the role of basic medium on the catalytic performance of catalyst in the direct reduction process. Thus, the effect of $\mathrm{NaOH}$ concentration on the catalytic activity of $5 \mathrm{Ni}-\mathrm{K}$ catalyst in conversion of PNP to PAP was studied as depicted in Fig. 7. It can be noted that the increase in $\mathrm{NaOH}$ concentration leads to decrease in the required time for completing the conversion of PNP to PAP compounds. Further increase of $\mathrm{NaOH}$ more than $2 \mathrm{~g}$ causes a drastic increase in the time needed for complete conversion. This finding may be attributed to the decrease in the mobility of reactants with increasing in $\mathrm{NaOH}$ concentration, and, hence, the reaction takes more time for complete conversion.

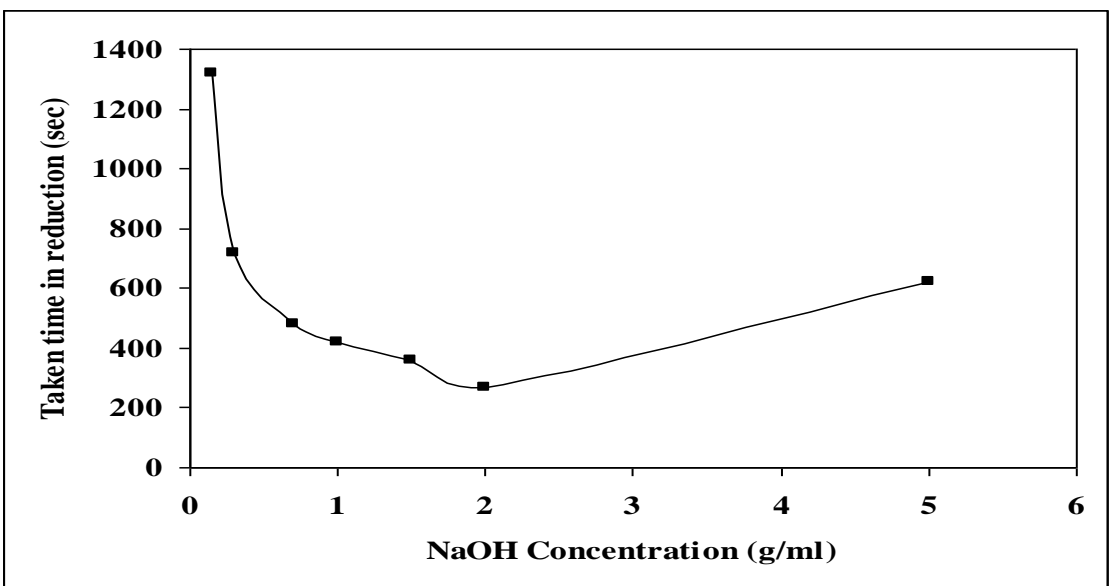

Fig. 7. Effect of $\mathrm{NaOH}$ dose on the conversion of PNP into PAP.

\section{Effect of ammonia concentration}

Figure 8 shows the effect of ammonia on the catalytic activity of $5 \% \mathrm{Ni}-\mathrm{K}$ for reduction of PNP. It was noted that as the concentration of ammonia increases from 0 to $25 \%$, the time required for complete reduction of PNP decreases from $430 \mathrm{sec}$ to $55 \mathrm{sec}$ (the decrease equal $\sim 87.3 \%$ ). It is a surprising result, where it was expected that the activity of catalyst decreased with addition of ammonia to reaction mixture as reported previously ${ }^{(2)}$. However, the interpretation of this finding may be attributed to generation of new active species on the nano-nickel catalyst, i.e., upon heating the reacting mixture, the adsorbed ammonia was released leaving more active centers locate on the catalyst and consequently the activity increased under successive addition of ammonium hydroxide. 


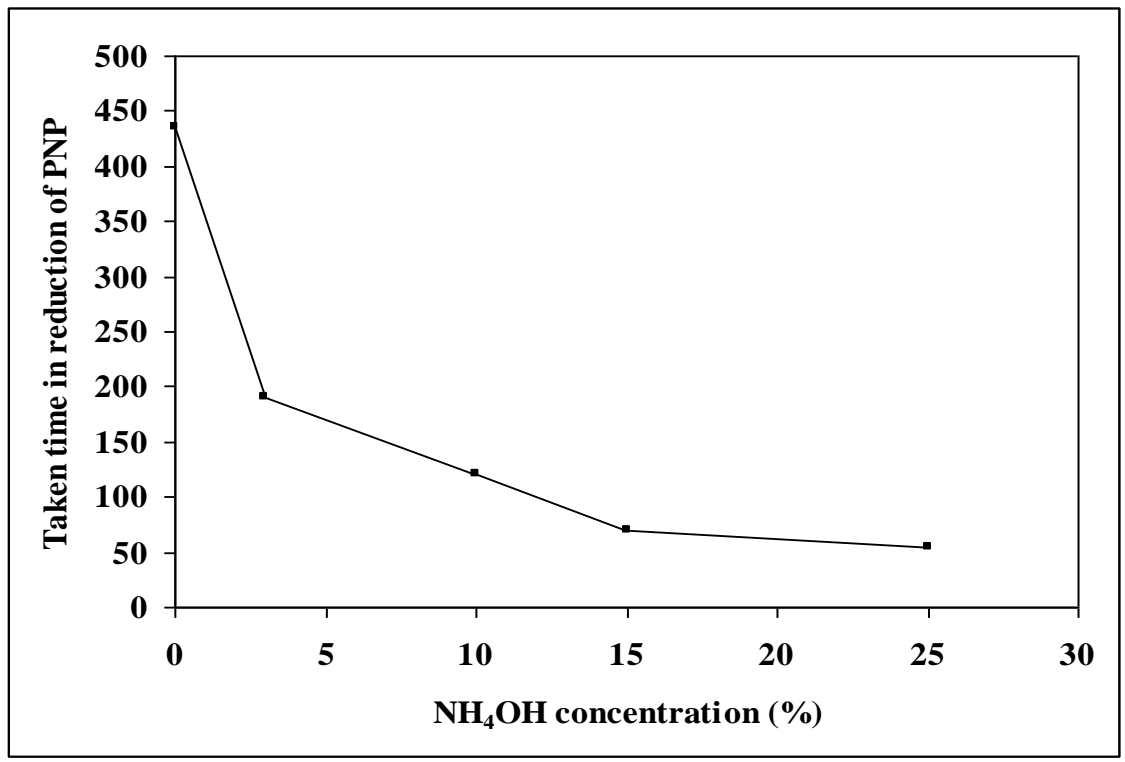

Fig.8. Effect of $\mathrm{NH}_{4} \mathrm{OH}$ concentration on the conversion of PNP into PAP.

\section{Conclusions}

A series of nano nickel catalyst supported on Egyptian kaolin as a support was prepared. The reduction process of nickel was performed using hydrazine hydrate as hydrogen donor. These catalysts were used for reduction of $\mathrm{p}$ nitrophenol to $\mathrm{p}$-aminophenol in the presence of hydrazine hydrate as hydrogen donor at elevated temperature $80^{\circ} \mathrm{C}$.

The increase of nickel on the support leads to an increase in the catalyst activity passing throw maximum activity when the nickel content was $5 \%$, which shows minimum time for complete reduction of $\mathrm{p}$-nitrophenol.

The increase of the weight of the catalyst from $0.2 \mathrm{~g}$ to $1 \mathrm{~g}$ decreases sharply the time for complete reduction from $30 \mathrm{~min}$ to $3.66 \mathrm{~min}$. Therefore, one gram catalyst was considered as optimum catalyst weight for complete conversion of p-nitrophenol.

It was found that the increase of added sodium hydroxide leads to a detectable decrease in the time taken for complete reduction of p-nitrophenol. Maximum effect was observed at concentration $2 \mathrm{~g} \mathrm{NaOH}$. Further increase led to increase in the time needed for the reduction. This was attributed to the decrease in the mobility of the reactants to be in contact with the catalyst.

The addition of a certain amount of ammonia to the reduced catalyst before addition of reactants leads to decrease the time needed for reduction of $\mathrm{p}$ -

Egypt. J. Chem. 55, No. 6 (2012) 
nitrophenol. The increase in the concentration of the added ammonia showed a noticeable decrease in the time taken for complete reduction. This can be explained by the fact that the heating of the reacting mixture that leads to release of adsorbed on nickel ammonia leaving more active sites for catalysis.

\section{References}

1. Zheng, H.G., Liang, J.H., Zeng, J.H. and Qian, Y.T., Preparation of nickel nanopowders in ethanol-water system (EWS). Mater. Res. Bull. 36, 947-952 (2001).

2. Du, Y., Chen, H., Chen, R. and Xu N., Poisoning effect of some nitrogen compounds on nano-sized nickel catalysts in $p$-nitrophenol hydrogenation. Chem. Eng. J. 125, 9 14 (2006).

3. Gao, J.Z., Guan, F., Zhao, Y.C., Yang, W., Ma, Y.J., Lu, X.Q., Hou, J.G. and Kang J.W., Preparation of ultrafine nickel powder and its catalytic dehydrogenation activity. Mater. Chem. Phys. 71, 215-219 (2001).

4. Selim M.M., Abd EI Maksod, I.H. and Saleh T.S., The use of nano supported nickel catalyst in reduction of p-nitrophenol using hydrazine hydrate as hydrogen donor. Egypt. J. Chem. 52 (4), 491-506 (2009).

5. Liu, H., Deng, J. and Li W., Synthesis of nickel nanoparticles supported on boehmite for selective hydrogenation of p-nitrophenol and p-chloronitrobenzene. Catal. Lett. 137, 261-266 (2010).

6. Chen, R.Z., Du, Y., Chen, C.L., Xing, W.H., Xu, N.P. Chen, C.X. and Zhang, Z.L., Comparative study on catalytic activity and stability of nano-sized nickel and Raney nickel. J. Chem. Ind. Eng. 54,704-706 (2003).

7. Du, Y. and Chen, H., Chen, R. and Xu, N., Synthesis of p-aminophenol from pnitrophenol over nano-sized nickel catalysts. Applied Catalysis A: General 277, 259264 (2004).

8. Du Y. and Chen R., Effect of nickel particle size on alumina supported nickel catalysts for p-nitrophenol hydrogenation. Chem. Biochem. Eng. Q. 21(3), 251-255 (2007).

9. Kapoor, S., Salunke, H.G., Tripathi, A.K., Kulshreshtha, S.K. and Mittal, J.P., Radiolytic preparation and catalytic properties of nanophase nickel metal particles. Mater. Res. Bull. 35, 143-148 (2000).

10. Rizhi, C., Yan, D., Weihong, X. and Nanping, X., Effect of alumina particle size on $\mathrm{Ni} / \mathrm{A}_{2} \mathrm{O}_{3}$ catalysts for p-nitrophenol hydrogenation. Chin. J. Chem. Eng. 15(6), 884888 (2007).

11. Yun, K.S. and Cho, B.W., Process for preparing para-aminophenol. US Pat. 5066369 (1991). 
12. Rode, C.V., Vaidya, M.J. and Chaudhari, R.V., Synthesis of p-aminophenol by catalytic hydrogenation of nitrobenzene. Org. Process Res. Dev. 3, 465-470 (1999).

13. Vaidya, M.J., Kulkami, S.M. and Chaudhari R.V., Synthesis of p-aminophenol by catalytic hydrogenation of p-nitrophenol. Org. Process Res. Dev. 7, 202-208 (2003).

14. Komatsu, T. and Hirose, T., Gas phase synthesis of para-aminophenol from nitrobenzene on Pt/ zeolite catalysts. Appl. Catal. A Gen. 276, 95-102 (2004).

15. Nishimura, S., Handbook of Heterogeneous Catalytic Hydrogenation for Organic Synthesis, Wiley, New York, (2001).

Received 3/4/ 2013;

accepted 22/4/2013)

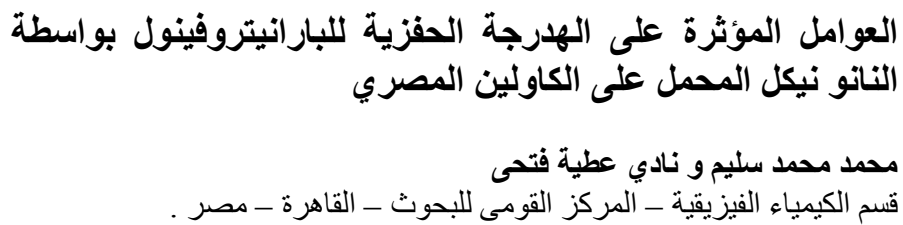

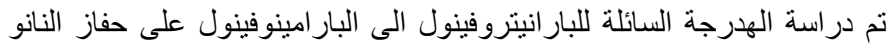

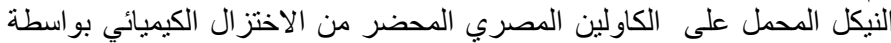

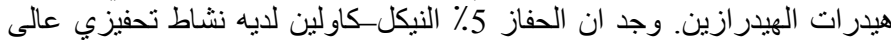

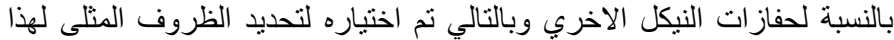

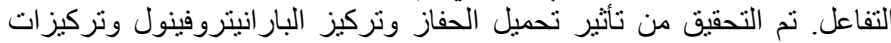

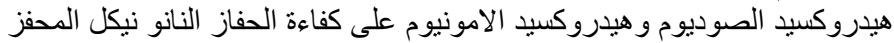

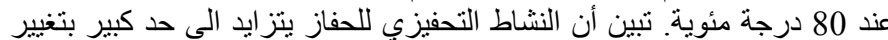

تركيز هيدروكسيد الصوديوم وهيدة ان هيدروكسيد الامونيوم خلال تفاعل الهدرجة.

Egypt. J. Chem. 55, No. 6 (2012) 